\title{
Symplectic Approach to the Theory of Quantized Fields. I*
}

\author{
P. L. García and A. Pérez-Rendón \\ Departamento de Algebra y Fundamentos \\ Facultad de Ciencias, Universidad de Barcelona, España
}

Received December 28, 1968

\begin{abstract}
Our aim in this paper the first one of a series concerned with the problem of field quantization starting from the symplectic structure underlying the classical theory, is to build up the variational theory necesary to all further constructions. The basic notions are the vertical bundle $\bar{B}$ and the structure 1-form $\theta$ used to define the generalized infinitesimal contact transformation which allows us to state and solve the variational problem related to field physics. Giving a system of modulevalued differential forms of different degree on the vertical bundle which solutions are the stationary cross sections is the main result in the paper. In this scheme the Euler-Lagrange classical equations are the expressions induced by such a system of differential forms on any cross section of the vertical bundle. This gives us a complete linearization of the Euler-Lagrange equations and, starting from it, a natural globalization of these equations. Finally, the notion of variational problem invariant by a Lie group is defined in this scheme, Noether's theorem related to such invariant problem is formulated and an intrinsic version of the so-called Noether invariants of classical variational calculus is obtained.
\end{abstract}

\section{Introduction}

The study of the symplectic structures that can be associated with classical fields in a natural way becomes more and more fascinating every day, on account of the important application done in recent years of such structures to the problem of field quantization (see, for instance, [6], [7] and [8]). This study is, on the other hand, important by itself, since it makes definitely clear the field dynamics as it was done some years ago with the ordinary analytic dynamics (see, for instance, [1] and [5]).

The first question to deal with is to decide which symplectic structure must be associated with a given classical field. I. SEgac has studied that problem in detail in the case of a scalar field on Minkowski's space-time defined by a non-linear hyperbolic differential equation in partial derivatives. Starting from the manifold of solutions of the field equation, he

* This work has been realized in the Seminar of Mathematical Physics, directed by Professor J. SANcho, in the Faculty of Science at the University of Barcelona (Spain). 
defines on the former a symplectic geometry by means of the propagator associated with the latter. In that way we meet the theory of propagators related to a hyperbolic differential equation in partial derivatives. That question has actually been studied by LichNERowicz using LERAY's general theory (see, for instance [4]). As far as we are concerned, we think that the real problem is in the variational theory; therefore, we have founded our proceedings in an intrinsic and global analysis of the variational problems which appear in field physics. The study of analytic dynamics from that point of view furnishes the leading idea of the adequate generalization.

Throughout this paper we are exclusively concerned with the variational problem. The steps and fundamental results are the following ones:

We start from a bundle $B$, direct product $V \times F$ of an $n$-dimensional differentiable manifold $V$ - which is assumed to be homeomorphic to the $n$-dimensional euclidean space, to avoid unnecessary complications and an $m$-dimensional differentiable manifold $F$. In the usual examples $V$ is Minkowski's space-time $V_{4}$, while the points of $B$ have the physical meaning of the possible vibration states of the field at every point of $V_{4}$. Usually, $F$ is assumed to be a vector space; however, we shall not restrict ourselves to such a simple hypothesis since then several interesting situations, such as those offered by analytic dynamics - which is the case when $V$ is the real line - when the configuration space is a Riemannian manifold, would be excluded. The theory could also be developed with slight changes, for an arbitrary bundle $B$. As a matter of fact, we don't care about this here, since in our opinion such generality is empty of physical meaning.

In our terminology the configurations of the field on $V$ are defined by the cross sections of $B$. If $s$ is one of such configurations and $X$ is a point of $V$, we have, for every tangent vector $D_{X} \in T_{X}(V)$, the tangent vector $(d s)_{X} D \in T_{s(X)}(B)$ which gives us, in a first approximation, a measure of the change endured by the configuration $s$ of the field when an infinitesimal movement from the point $X$, in the direction $D_{X}$ is undertaken. Then the linear injection $(d s)_{X}$ can be regarded as a first approximation of the configuration $s$ of the field in the neighbourhood of the point $X$. A vectorial bundle structure over $B$ can be defined on the set $\bar{B}$ of all those first approximations. That bundle - the vertical bundle - is the basic tool of our theory.

An $M$-valued 1-form $\theta$ - the structure 1 -form - where $M$ is an $A_{\bar{B}^{-}}$ module $-A_{\bar{B}}$ is the algebra of differentiable functions on $\bar{B}$ - can be canonically defined on the bundle $\bar{B}$. We are constricted to such a modulevaluation as we started from a differentiable manifold $F$. In the special case when $F$ is the number space $R^{m}$, the $A_{\bar{B}}$-module $M$ is $A_{\bar{B}}$ and, consequently, the 1 -form $\theta$ turns into $m$ ordinary 1 -forms $\theta j$, whence the 
problem becomes much simpler. Starting from the structure 1-form $\theta$ we define the fundamental notion of generalized infinitesimal contact transformation (g.i.c.t.). A g.i.c.t. is a vector field $\bar{D}$ on $\bar{B}$ such that for every derivation law $\nabla$ on $M$ we have

$$
L_{D} \theta=\Phi \circ \theta
$$

where $\Phi \in \operatorname{Hom}_{\boldsymbol{A}_{\bar{B}}}(M, M)$, the dot product $\circ$ is the one induced by the usual bilinear product $\operatorname{Hom}_{A_{\bar{B}}}(M, M) \times M \rightarrow M$, and $L_{D}$ is the Lie derivation with respect to $\bar{D}$. In the particular case where $F=R^{m}$, the condition $L_{D} \theta=\Phi \circ \theta$ is equivalent to the ordinary $m$ conditions $L_{D} \theta_{j}=\sum_{i} \Phi_{i j} \theta_{i}$. In other words, the one-parameter group associated to $D$ leaves the Pfaff system $\left[\theta_{1} \ldots \theta_{m}\right]$ invariant.

Starting from the notion of g.i.c.t. we can construct the variational calculus.

The Lagrangian density is defined by an $n$-form $\mathscr{L} \omega$ on the vertical bundle $\bar{B}$ where $\mathscr{L}$ is a real function on $\bar{B}$ and $\omega$ is a volume element defined on the base manifold $V$. The fundamental idea is now to call stationary a cross section $\bar{s}: V \rightarrow \bar{B}$ solution of the structure 1 -form $\theta$ and such that for every g.i.c.t. we have

$$
\int_{\bar{s}(V)} L_{\bar{D}}(\mathscr{L} \omega)=0
$$

Actually, the most important result in this paper is the following: to each linear connection $\nabla$ on the differentiable manifold $F$ can be canonically associated a system of module-valued differential forms of different degree on the vertical bundle whose solutions are the stationary cross sections of our variational problem. This system induces locally on the cross sections of $B$ a set of expressions that are precisely the EulerLagrange equations of the classical variational calculus. In this way we get an intrinsic characterization of such classical equations and therefore a natural way of globalizing them.

We conclude this paper by defining the notion of a variational problem invariant by a Lie group and giving an adequate formulation of Noether's theorem related to such invariant problems. As in the case of the Euler-Lagrange equations, the so-called Noether invariants in this paper are, in fact, $(n-1)$-forms on the vertical bundle which induce on a cross section $\bar{s}$ of $B$ the classical expressions of such invariants working in a good coordinate system of course.

Lately, Trautman has studied the variational theory of classical fields from a point of view somewhat analogous to ours.

In our terminology, the author starts from a bundle $B=V \times F$ direct product of $V=R^{n}$ and $F=R^{N}$ and he defines the variational 
theory on the bundle $\bar{B}$ of the 1 -jets of the cross sections of $\bar{B}$. Without regarding the generality of the initial bundle $B$, which carries non trivials global problems, our method differs essentially from that of TRAUTMAN in the introduction of the structure 1 -form $\theta$. This has allowed us to obtain the results we mentioned before and that will later be used to build up all the dynamic notions associated with a field (simplectic geometry, Poisson bracket, Hamilton equations, etc.) according to what we stated at the beginning of the introduction.

\section{Vertical Bundle $\bar{B}$ and Strueture 1-Form $\boldsymbol{\theta}$}

Let $V$ be a differentiable $n$-dimensional manifold homeomorphic to an euclidean space of dimension $n, F$ a differentiable $m$-dimensional manifold and $B$ the direct product of both manifolds. Denote $\pi$ and $\pi^{\prime}$ the canonical projections of $B$ onto $V$ and $F$, respectively.

Let $T(V), T(F)$ and $T(B)$ be the tangent bundles [3] of the manifolds $V, F$ and $B$ and call $d \pi, d \pi^{\prime}$ the homomorphic mappings of $T(B)$ onto $T(V)$ and $T(F)$ induced by the canonical projections $\pi$ and $\pi^{\prime}$, respectively.

Definition 1.1. If $T(V)_{B}, T(F)_{B}$ are the pull-backs [3] of $T(V), T(F)$ over $B$, we say that $\bar{B}=\operatorname{Hom}\left(T(V)_{B}, T(F)_{B}\right)$ is the vertical bundle over $B$. We denote $\bar{\pi}$ the canonical projection of $\bar{B}$ onto $B$.

Notations. From now on, we shall denote:

with

$$
P=\bar{\pi} \bar{P} ; P=(X, Y)
$$

$$
\begin{gathered}
X \in V ; Y \in F ; \\
D_{P}=d \bar{\pi}\left(\bar{D}_{\bar{P}}\right) \quad \text { and } \quad D_{X}^{\prime}=d \pi\left(D_{P}\right)
\end{gathered}
$$

where $\bar{P}$ is an arbitrary point of $\bar{B}$ and $\bar{D}_{\bar{P}}$ any element of the tangent space $T_{\bar{P}}(\bar{B})$ of $\bar{B}$ at $\bar{P}$.

Lemma 1.1. We can establish a one-to-one correspondance between the points $\bar{P}$ of $\bar{B}$ and the homomorphisms $i_{\bar{P}}$ that map the tangent space $T_{X}(V)$ of $V$ at $X$ into the tangent space $T_{P}(B)$ of $B$ at $P$, such that

$$
T_{P}(B)=i_{\bar{P}}\left(T_{X}(V)\right) \oplus T_{P}(F)
$$

where $T_{P}(F)$ is the fiber of $T(F)_{B}$ at $P$.

Proof. Since $B$ is the direct product of manifolds $V$ and $F, T(B)$ is the Whitney sum [3] of bundles $T(V)_{B}$ and $T(F)_{B}$; we can then assign to every point $\widetilde{P}$ a homomorphism $i_{\bar{P}}$ of $T_{X}(V)$ into $T_{P}(B)$ in the following way:

If $\bar{P}=\left(P, \sigma_{\bar{P}}\right)$, where $\sigma_{\bar{P}} \in \underset{R}{\operatorname{Hom}}\left(T_{P}(V), T_{P}(F)\right)$, then $i_{\bar{P}}=\sigma_{\bar{P}}+I$, where $I$ is the identity on $T_{P}(V)$. 
Moreover, $T_{P}(B)=i_{\bar{P}}\left(T_{X}(V)\right) \oplus T_{P}(F)$.

Let $s$ be a regular cross section of bundle $B$ and let $P$ be a point of the submanifold $s(V)$ of $B$.

Definition 1.2. The linear mapping of $T_{P}(B)$ onto $T_{P}(F)$ defined as follows:

$$
d^{v} s_{P}(D)=D_{P}-(d s)_{X} D^{\prime}
$$

is called vertical differential of $s$ at $P$.

It follows immediatly from the definition that the sequence

$$
0 \longrightarrow T_{X}(V) \stackrel{d s}{\longrightarrow} T_{P}(B) \stackrel{d^{v} s}{\longrightarrow} T_{P}(F) \longrightarrow 0
$$

is exact and, if $s_{1}, s_{2}$ are two cross sections of $B$ such that

$$
\left(d s_{1}\right)_{X}=\left(d s_{2}\right)_{X}, \text { then } \quad\left(d^{v} s_{1}\right)_{P}=\left(d^{v} s_{2}\right)_{P} .
$$

This property suggests the following

Definition 1.3. If $\bar{P}$ is a point of the vertical bundle $\bar{B}, s$ a regular cross section of $B$ such that $\bar{P}=\left(s(X), d s_{X}\right)$ and $\theta_{\bar{P}}$ is the linear mapping of $T_{\bar{P}}(\bar{B})$ in $T_{P}(F)$ :

$$
\theta_{\bar{P}}(\bar{D})=d^{v} s_{P}(D)
$$

then $\theta_{\bar{P}}$ is called structure 1 -form at the point $\bar{P}$ of the vertical bundle $\bar{B}$.

The embedding $\bar{s}$ of $V$ into $\bar{B}$ such that

$$
\bar{s}(X)=\left(s(X), d s_{X}\right)
$$

is called canonical lift of $s$ to $\bar{B}$; we can see that the form $\theta$ assigns the vector field $d^{v} s(D)$ with support in $s(V)$ to every vector field $\bar{D}$ with support in the submanifold $\bar{s}(V)$ of $\bar{B}$. A Lemma follows immediately from these definitions :

Lemma 1.2. The structure form verifies the following properties:

a) $\theta$ vanishes on the tangent vector fields to the fibers of $\bar{B}$.

b) The map induced by $\theta$ on the submanifold $\bar{s}(V)$ of $\bar{B}$ vanishes.

Theorem 1.1. Let $\left(x_{1} \ldots, x_{n}\right)$ be a coordinate system on the manifold $V$ and let $U$ be an open set in $F$ with local coordinates $\left(z_{1} \ldots, z_{m}\right):$ We can find $m \cdot n$ functions $p_{i j}(i=1 \ldots, n ; j=1 \ldots, m)$ on $\bar{\pi}^{-1}(V \times U)$ such that:

a) $\left(x_{i}, z_{j}, p_{i j}\right)$ is a local coordinate system of $\bar{B}$ in $\bar{\pi}^{-1}(V \times U)$.

b) The 1-form $\theta$ can be written in that coordinate system in the following way:

$$
\theta_{\bar{P}}(\bar{D})=\sum_{j}\left[\left(d z_{j}-\sum_{i} p_{i j} d x_{i}\right)_{\bar{P}} \bar{D}\right]\left(\frac{\partial}{\partial z_{j}}\right)_{P}
$$

for every $\bar{D}_{\bar{P}} \in T_{\bar{P}}(\bar{B})$ and $\bar{P} \in \bar{\pi}^{-1}(V \times U)$.

Proof. Let $p_{i j}$ be functions on $\bar{\pi}^{-1}(V \times U)$ defined by

$$
p_{i j}(\bar{P})=\left[\sigma_{\bar{P}}\left(\frac{\partial}{\partial x_{i}}\right)\right] z_{j},
$$

where $\bar{P}=\left(P, \sigma_{\bar{P}}\right)$ and $\sigma_{\bar{P}} \in \underset{R}{\operatorname{Hom}}\left(T_{P}(V), T_{P}(F)\right)$. 
By the definition of the vertical bundle $\bar{B}$, the functions $\left(x_{i}, z_{j}, p_{i j}\right)$ are a local coordinate system of $\bar{B}$ in $\bar{\pi}^{-1}(V \times U)$.

On the other hand, if we apply $\theta_{\bar{P}}$ to the derivations

we have:

$$
\frac{\partial}{\partial x_{j}}, \quad \frac{\partial}{\partial z_{j}}, \quad \frac{\partial}{\partial p_{i j}},
$$

$$
\begin{aligned}
\theta_{\bar{P}}\left(\frac{\partial}{\partial x_{i}}\right)=d^{v} s_{P}\left(\frac{\partial}{\partial x_{i}}\right) & =\left(\frac{\partial}{\partial x_{i}}\right)_{P}-d s_{X}\left(\frac{\partial}{\partial x_{i}}\right)=-\sigma_{\bar{P}}\left(\frac{\partial}{\partial x_{i}}\right) \\
& =-\left(\sum_{j} p_{i j} \frac{\partial}{\partial z_{j}}\right)_{P},
\end{aligned}
$$

since Lemma 1.1. gives $d s_{X}=\sigma_{\bar{P}}+I$, where $d s_{X}$ is the homomorphism of $T_{X}(V)$ into $T_{P}(B)$ associated to the point $\bar{P}$.

and, finally:

$$
\theta_{\bar{P}}\left(\frac{\partial}{\partial z_{j}}\right)=d^{v} s_{P}\left(\frac{\partial}{\partial z_{j}}\right)=\left(\frac{\partial}{\partial z_{j}}\right)_{P}
$$

which completes the proof.

$$
\theta_{\bar{P}}\left(\frac{\partial}{\partial p_{i j}}\right)=0
$$

Definition 1.4. A local coordinate system of $\bar{B}$ in a neighbourhood $U$ of $\bar{B}$ is called canonical if it verifies the conditions of theorem 1.1.

\section{Module - Valued Tensor Calculus}

Let $M$ be the set of differentiable mappings $f$ of $\bar{B}$ in $T(F)_{B}$ such that

where $D_{P}^{v} \in T_{P}(F)$.

$$
\bar{P} \stackrel{f}{\longrightarrow} D_{P}^{v}
$$

Lemma 2.1. The set $M$ is a $A_{\bar{B}}$-module, where $A_{\bar{B}}$ is the algebra of functions of class $C^{\infty}$ defined on the manifold $\bar{B}$.

Proof. We give the following definitions of sum and product by a function $g$ of $A_{\bar{B}}$ :

a) $\bar{P} \stackrel{f_{1}+f_{2}}{\longrightarrow}\left(D_{1}^{v}+D_{2}^{v}\right)_{P}$

b) $\bar{P} \stackrel{g \cdot f}{\longrightarrow} g(\bar{P}) D_{P}^{v}$

The rest is a straight-forward matter.

In a similar way, we can build the $A_{\bar{B}}$-module $M^{*}$ of differentiable mappings $f^{*}$ of $\bar{B}$ into the cotangent bundle $T^{*}(F)_{B}$ such that

$$
\bar{P} \stackrel{f^{*}}{\longrightarrow} \omega_{P}^{v}
$$

Since most of the definitions and properties of the $A_{\bar{B}}$-module $M$ that we give hereafter are also verified by $M^{*}$, we shall prove our statements only for $M$ and we shall understand that they remain valid for $M^{*}$ too.

Let $\{\bar{D}\}$ be the $A_{\bar{B}}$-module of vector fields of $\bar{B}$ and $\{\bar{\omega}\}$ its dual. We can now define $M$-valued tensors on $\bar{B}$ in the following way: 
Every $p+q$-linear mapping $T_{p}^{q}$ of $\{\bar{D}\}^{p} \times\{\bar{\omega}\}^{q}$ in $M$ is called a $p$ covariant, $q$-contravariant $M$-valued tensor.

We introduce those new tensors because the structure form $\theta$ is a 1-covariant $M$-valued tensor.

For this reason we shall give hereafter the most important results of this module-valued tensor calculus; the reader may check the proofs in KoszuL's lectures [2].

Let $K$ be a commutative field, $A$ a commutative and associative algebra and $M$ an $A$-module. Let $\{D\}$ be the $A$-module of derivations of $A$ into $A$ over $K$ and let $\{\omega\}$ be its dual.

Definition 2.1. An $M$-valued tensor $T_{p}^{q}$ (p-covariant, $q$-contravariant) on $A$ is a $p+q$-linear mapping of $\{D\}^{p} \times\{\omega\}^{q}$ in $M$.

As in the ordinary case, the set of all tensors $\left\{T_{p}^{q}\right\}$ with fixed indices $p, q$ is an $A$-module with respect to the natural laws of addition of tensors and multiplication by a scalar.

Definition 2.2. Let $M_{1}, M_{2} M_{3}$, be three $A$-modules with a bilinear product $M_{1} \times M_{2} \rightarrow M_{3}$ that we shall denote $\left(e, e^{\prime}\right) \rightarrow e e^{\prime}$. If $T_{p}^{q}$ is an $M_{1^{-}}$ valued tensor and $T_{r}^{8}$ an $M_{2}$-valued tensor, we say that the $M_{3}$-valued tensor $T_{p}^{q} \otimes T_{r}^{s}$ is the tensor product of both, with $T_{p}^{q} \otimes T_{r}^{s}$ defined as follows: $T_{p}^{q} \otimes T_{r}^{s}\left(D_{1} \ldots D_{p} . D_{1}^{\prime} \ldots D_{r}^{\prime}, \omega_{1} \ldots \omega_{q}, \omega_{1}^{\prime} \ldots \omega_{s}^{\prime}\right)$ $=T_{p}^{q}\left(D_{1} \ldots D_{p}, \omega_{1} \ldots \omega_{q}\right) T_{r}^{s}\left(D_{1}^{\prime} \ldots D_{r}^{\prime}, \omega_{1}^{\prime} \ldots \omega_{s}^{\prime}\right)$.

\section{Three very Important Examples}

a) Let $M_{1}=A ; M_{2}=M_{3}=M$ and let $A \times M \rightarrow M$ be the product defined by the module structure. Then the tensor product of an ordinary tensor and an $M$-valued tensor is an $M$-valued tensor.

b) Let $M_{1}=M, M_{2}=M^{*}$ (dual of $M$ ), $M_{3}=A$ and let $M \times M^{*} \rightarrow A$ be the mapping $(e, \omega) \rightarrow \omega(e)$. The tensor product of an $M$-valued tensor and an $M^{*}$-valued tensor is an ordinary tensor.

c) Let $M_{1}=\underset{A}{\operatorname{Hom}}(M, M), M_{2}=M_{3}=M$ and let $\underset{A}{\operatorname{Hom}}(M, M)$ $\times M \rightarrow M$ by the ${ }^{A}$ map $(\tau, e) \rightarrow \tau(e)$. Then the tensor product of an $\underset{A}{H}(M, M)$-valued tensor and an $M$-valued tensor is an $M$-valued tensor.

These three examples of tensor products will be often employed throughout the paper.

The Lie derivation on module-valued tensors may be introduced in a natural way when we have previously defined a derivation law in that module.

Definition 2.3. A derivation law in an $A$-module $M$ is a mapping $V$ of the $A$-module $\{D\}$ in the homomorphisms $\underset{K}{\mathrm{Hom}}(M, M)$ of $M$ :

$$
D \stackrel{\nabla}{\longrightarrow} D^{\nabla} \in \underset{\boldsymbol{K}}{\operatorname{Hom}}(M, M)
$$


such that

a) $\left(D_{1}+D_{2}\right)^{\nabla}=D_{1}^{\nabla}+D_{2}^{\nabla}$

b) $\left(a D_{1}\right)^{\nabla}=a \cdot D_{1}^{\nabla^{1}}$

c) $D_{1}^{\nabla}(a e)=\left(D_{1} a\right) e+a D_{1}^{V} e$ where $a \in A, e \in M, D_{1}, D_{2} \in\{D\}$.

For instance, if we consider $A$ as an $A$-module, then $V$ defined by $D^{\nabla} a=D a$ is a derivation law that we shall call canonical derivation in $A$.

The Lie derivation is introduced as follows:

Definition 2.4. Let $\nabla$ be a derivation law in the $A$-module $M$ and $\left\{T_{p}^{q}\right\}$ the $A$-module of $p$-covariant, $q$-contravariant $M$-valued tensors on $A$. We shall say that the map $L$ of $\{D\}$ in $\underset{K}{\operatorname{Hom}}\left(\left\{T_{p}^{q}\right\},\left\{T_{p}^{q}\right\}\right)$ is a Lie derivation in $\left\{T_{p}^{q}\right\}$ if:

$$
\begin{aligned}
\left(L_{D} T_{p}^{q}\right)\left(D_{1} \ldots, D_{p}, \omega_{1} \ldots, \omega_{p}\right)=D^{\nabla} T_{p}^{q}\left(D_{1} \ldots D_{p}, \omega_{1} \ldots, \omega_{q}\right) \\
-\sum_{i} T_{p}^{q}\left(D_{1} \ldots,\left[D, D_{i}\right], \ldots, D_{p}, \omega_{1} \ldots, \omega_{q}\right) \\
-\sum_{i} T_{p}^{q}\left(D_{1} \ldots, D_{p}, \omega_{1} \ldots, L_{D} \omega_{i} \ldots, \omega_{q}\right) .
\end{aligned}
$$

The tensor $L_{D} T_{p}^{q}$ is called the Lie derivative of $T_{p}^{q}$ with respect to $D$.

The Lie derivation verifies the following properties:

$$
\begin{aligned}
L_{D}\left(T+T^{\prime}\right) & =L_{D} T+L_{D} T^{\prime} \\
L_{D}(a T) & =(D a) T+a L_{D} T \\
L_{D+D^{\prime}}(T) & =L_{D} T+L_{D^{\prime}} T \\
L_{\lambda D}(T) & =\lambda L_{D} T
\end{aligned}
$$

where $D, D^{\prime} \in\{D\}, \quad T, T^{\prime} \in\left\{T_{p}^{q}\right\}, \quad \lambda \in K$.

We shall now see the relationship between the Lie derivation and the tensor product:

Let $M_{1}, M_{2}, M_{3}$ be three $A$-modules with a bilinear product $M_{1} \times M_{2} \rightarrow M_{3}$, and let $\nabla_{1}, \nabla_{2}, \nabla_{3}$ be three derivation laws given in $M_{1}, M_{2}, M_{3}$ respectively. We say that the bilinear product is compatible with the derivation laws when

$$
D^{V_{3}}\left(e e^{\prime}\right)=\left(D^{V_{1}} e\right) e^{\prime}+e\left(D^{V_{2}} e^{\prime}\right)
$$

where $D \in\{D\}, \quad e \in M_{1}, \quad e^{\prime} \in M_{2}$.

For instance, if we define the canonical derivation law in $A$, the derivation law $\left(D^{\nabla} \omega\right) e=D(\omega(e))-\omega\left(D^{\nabla} e\right)$ in $M^{*}$, and the derivation law $\left(D^{\nabla} \tau\right) e=D^{\nabla}(\tau(e))-\tau\left(D^{\nabla} e\right)\left(\right.$ where $\left.\tau \in \operatorname{Hom}_{A}(M, M)\right)$ in $\mathrm{Hom}_{A}(M, M)$ then the three bilinear products $A \times \underset{A}{\rightarrow} \rightarrow M, M \times M^{*} \rightarrow A$ and Hom $(M, M) \times M \rightarrow M$ are compatible with the respective laws. 
Let now $T_{p}^{q}$ be an $M_{1}$-valued tensor and $T_{r}^{s}$ an $M_{2}$-valued tensor and assume that the bilinear product $M_{1} \times M_{2} \rightarrow M_{3}$ is compatible with the derivation laws $\nabla_{1}, \nabla_{2}, \nabla_{3}$. Then for every $D \in\{D\}$ we have

$$
L_{D}\left(T_{p}^{q} \otimes T_{r}^{s}\right)=L_{D} T_{p}^{q} \otimes T_{r}^{s}+T_{p}^{q} \otimes L_{D} T_{r}^{s}
$$

We shall now deal with the differential forms following the same method.

Definition 2.5. $A M$-valued p-form $\omega_{p}$ on $A$ is a p-covariant skewsymmetric, $M$-valued tensor.

The set $\left\{\omega_{p}\right\}$ of all $p$-forms is a submodule of the $A$-module $\left\{T_{p}\right\}$ of $p$-covariant tensors.

Definition 2.6. Let $M_{1}, M_{2}, M_{3}$ be three A-modules with a bilinear product $M_{1} \times M_{2} \rightarrow M_{3}$. Let $\omega_{p}$ be a $M_{1}$-valued form and $\omega_{q}$ a $M_{2^{-}}$ valued form. The $M_{3}$-valued form $\omega_{p} \wedge \omega_{q}$ given by:

$$
\begin{aligned}
\left(\omega_{p} \wedge \omega_{q}\right) & \left(D_{1} \ldots, D_{p+q}\right) \\
= & \frac{1}{(p+q) !} \sum\left(\text { sign of } s_{j k}\right) \omega_{p}\left(D_{j_{1}}, \ldots, D_{j_{p}}\right) \omega_{q}\left(D_{k_{1}}, \ldots, D_{k_{q}}\right),
\end{aligned}
$$

where $s_{j k}$ is the permutation $(1 \ldots, p+q) \rightarrow\left(j_{1} \ldots, j_{p}, k_{1} \ldots, k_{q}\right)$, is called the exterior product of forms $\omega_{p}, \omega_{q}$.

It follows immediately from the definition of Lie derivative of a tensor that the Lie derivative $L_{D} \omega$ of a form $\omega$ is also a form and that, if $M_{1}, M_{2}, M_{3}$ are three modules with derivation laws compatible with a bilinear product, $\omega_{p}$ a $M_{1}$-valued form and $\omega_{q}$ a $M_{2}$-valued from, then

$$
L_{D}\left(\omega_{p} \wedge \omega_{q}\right)=L_{D} \omega_{p} \wedge \omega_{q}+\omega_{p} \wedge L_{D} \omega_{q} .
$$

We can introduce the inner product and the exterior differentiation on module-valued forms in the same way as in the ordinary case.

For every $D \in\{D\}$, the homomorphism $i D:\left\{\omega_{p}\right\} \rightarrow\left\{\omega_{p-1}\right\}$ given by

$\left(i D . \omega_{\mathfrak{p}}\right)\left(D_{1} \ldots, D_{p-1}\right)=p \omega_{p}\left(D, D_{1} \ldots . D_{p-1}\right)(p>0) i D . \omega_{0}=0$

is called inner product $i D$. on the $A$-module $\left\{\omega_{p}\right\}$.

The inner product verifies the following properties:

$$
\begin{aligned}
L_{D} i D^{\prime} .+i D^{\prime} . L_{D} & =i\left[D, D^{\prime}\right] . \\
i(a D) . & =a i D . \\
(i D .)^{2} & =0 \\
i\left(D+D^{\prime}\right) . & =i D .+i D^{\prime} .
\end{aligned}
$$

where $a \in A$ and $D, D^{\prime} \in\{D\}$.

Let three modules $M_{1}, M_{2}, M_{3}$ be given, with derivation laws compatible with a bilinear product; if $\omega_{p}$ is an $M_{1}$-valued from and $\omega_{q}$ an $M_{2}$-valued form; then we have:

$$
i D .\left(\omega_{p} \wedge \omega_{q}\right)=i D \cdot \omega_{p} \wedge \omega_{q}+(-1)^{q} \omega_{p} \wedge i D \cdot \omega_{q} .
$$


The $K$-linear map of $\left\{\omega_{p}\right\}$ in $\left\{\omega_{p+1}\right\}$ given by

$$
\begin{aligned}
& d \omega_{p}\left(D_{1} \ldots, D_{p+1}\right) \\
& =\frac{1}{p+1} \sum_{i=1}^{p+1}(-1)^{i+1} D_{i}^{\Gamma}\left(\omega_{p}\left(D_{1} \ldots, \hat{D}_{i} \ldots, D_{p+1}\right)\right) \\
& \quad+\frac{1}{p+1} \sum_{1 \leqq i \leqq j \leqq p+1}(-1)^{i+j} \omega_{p}\left(\left[D_{i}, D_{j}\right], D_{1} \ldots, \hat{D}_{i} \ldots, \hat{D}_{j} \ldots D_{p}\right)
\end{aligned}
$$

where the symbol $\wedge$ on a term denotes the omission of that term, is called exterior differentiation on the $A$-module $\left\{\omega_{p}\right\}$.

Let three modules $M_{1}, M_{2}, M_{3}$ be given, with derivation laws compatible with a bilinear product; if $\omega_{p}$ is a $M_{1}$-valued form and $\omega_{q}$ a $M_{2}$-valued form, then we have:

$$
d\left(\omega_{p} \wedge \omega_{q}\right)=d \omega_{p} \wedge \omega_{q}+(-1)^{p} \omega_{p} \wedge d \omega_{q} .
$$

Inner product, exterior differentiation on Lie derivation are connected by an important formula:

$$
L_{D}=i D . d+d i D .
$$

It is wellknown that the exterior differentiation on the algebra of ordinary differential forms on a manifold verifies $d^{2}=0$. However, in the case of module-valued forms this is not true in general. Thus, if $e$ is an element of $M$, we have:

$$
\begin{aligned}
d^{2} e\left(D, D^{\prime}\right) & =D^{\nabla}\left(d e\left(D^{\prime}\right)\right)-D^{\prime \nabla}(d e(D))-d e\left(\left[D, D^{\prime}\right]\right) \\
& =D^{\nabla} D^{\prime \nabla} e-D^{\prime \nabla} D^{\nabla} e-\left[D, D^{\prime}\right]^{\nabla} e \neq 0 .
\end{aligned}
$$

If we write $K\left(D, D^{\prime}\right)=D^{\nabla} D^{\prime \nabla}-D^{\prime \nabla} D^{\nabla}-\left[D, D^{\prime}\right]^{\nabla}$ it is easy to check that $K\left(D, D^{\prime}\right)$ is an $A$-endomorphism of $M$ and, moreover:

$$
\begin{aligned}
K\left(D, D^{\prime}\right) & =-K\left(D^{\prime}, D\right) \\
K\left(D+D^{\prime}, D^{\prime \prime}\right) & =K\left(D, D^{\prime \prime}\right)+K\left(D^{\prime}, D^{\prime \prime}\right) \\
K\left(a D, D^{\prime}\right) & =a K\left(D, D^{\prime}\right)
\end{aligned}
$$

Then $K$ is an $\mathrm{Hom}_{A}(M, M)$-valued 2 -form on $A$.

Definition 2.7. The 2-form $K$ is called curvature form of the derivation law $\nabla$.

In the special case of $M=A$ and $\nabla$ being the canonical derivation law, we have:

$$
K\left(D, D^{\prime}\right) a=D D^{\prime} a-D^{\prime} D a-\left[D, D^{\prime}\right] a=0
$$

The following results (which proofs are in [2], ch-1) become of main importance in applications:

$$
\begin{aligned}
L_{D} L_{D^{\prime}} \omega-L_{D^{\prime}} L_{D} \omega & =L_{\left[D, D^{\prime}\right]} \omega+K\left(D, D^{\prime}\right) \omega \\
L_{D} d \omega-d L_{D} \omega & =(i D . K) \wedge \omega \\
d^{2} \omega & =K \wedge \omega ; \quad d K=0
\end{aligned}
$$

3 Commun. math. Phys., Vol.13 
$\wedge$ denotes here the exterior product induced by the bilinear product $\operatorname{Hom}(M, M) \times M \rightarrow M$.

${ }^{A}$ Finally, assume that $A$ is an algebra such that the $A$-module $\{D\}$ of its derivations is free of $k$ dimension; if that is the case, and if $M_{1}, M_{2}, M_{3}$ are three $A$-modules with a bilinear product $M_{1} \times M_{2} \rightarrow M_{3}, \bar{D}$ is an 1-contravariant $M_{1}$-valued tensor, and $\omega_{p}$ is an $M_{2}$-valued $p$-form, then we define the contraction of $\bar{D}$ and $\omega_{p}$ in the following way:

Let $\left(D_{j}, \ldots, D_{k}\right)$ be an arbitrary basis of the $A$-module $\{D\}$, and let $\left(\omega_{1}, \ldots, \omega_{k}\right)$ be the dual basis. We define the contraction of $\bar{D}$ an $\omega_{p}$ to be the $M_{3}$-valued $(p-1)$-form $i \bar{D} . \omega_{p}$ such that

$$
\left(i \bar{D} . \omega_{p}\right)\left(D_{1}^{\prime}, \ldots, D_{p-1}^{\prime}\right)=\sum_{i=1}^{k} \bar{D}\left(\omega_{i}\right) \omega_{p}\left(D_{i}, D_{1}^{\prime}, \ldots, D_{p-1}^{\prime}\right)
$$

for any $D_{1}^{\prime}, \ldots, D_{p-1}^{\prime} \in\{D\}$.

It is easy to check that the $(p-1)$-form thus defined does not depend on the choice of the basis $\left(D_{1}, \ldots, D_{k}\right)$ of $\{D\}$.

As we said at the beginning of this section, we are now interested in applying the tensor calculus thus far developed to the special case of $A$ being $A_{\bar{B}}$ and $M$ being the $A_{\bar{B}}$-module of differentiable maps of $\bar{B}$ in $T(F)_{B}$. If this is the case, we can define a derivation law on $M$ starting from a linear connection $V$ on $F$ in the following way:

Let $f$ be any element of $M$ and $\omega$ a 1-form in $F$; we may assign to both $f, \omega$ a function $g$ in $A_{\bar{B}}$ in the following way:

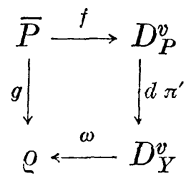

Definition 2.8. Let an element $f$ in $M$ and a derivation $\bar{D}$ in $\{\bar{D}\}$ be given, we define the derivative of $f$ with respect to $\bar{D}$, at the point $\bar{P}$, as the element $\bar{D}_{\bar{P}} f \in T_{P}(F)$ such that:

$$
\omega_{Y}\left(d \pi^{\prime}\left(\bar{D}_{\bar{P}} f\right)\right)=\bar{D}_{\bar{P}} g-\left(D^{\prime \prime \nabla} \omega\right)_{Y} D^{v}
$$

where $\omega$ is any 1-form in $F, D_{Y}^{\prime \prime}$ the projection of the derivation $D_{P}$ in $B$ on $F$ and $\nabla$ is the given linnear connection.

The element $\bar{D} f$ of $M$ that assigns to every point $\bar{P}$ in $\bar{B}$ the element $\bar{D}_{\bar{P}} f$ in $T_{P}(F)$ is called derivative of $f$ with respect to $\bar{D}$.

From now on, whenever we speak about a derivation law in the $A_{\bar{B}^{-}}$ module $M$, we are referring to this special type of derivation law defined by a linear connection $\nabla$ on $F$. Furthermore, we shall assume that the symmetry condition $D_{1}^{\nabla} D_{2}=D_{2}^{\nabla} D_{1}$ is verified by the linear connection.

We end this study on $M$-valued tensors on $\bar{B}$ with a last question: How to coordinate the vector space $\left\{T_{p}^{q}\right\}$ of $M$-valued, $p$-covariant, $q$-contravariant tensors. 
Lemma 2.2. $M$ is a m-dimensional locally free $A_{\bar{B}}$-module.

Proof: Let $U$ be any open set in $\bar{B}$ with canonical coordinates $\left(x_{i}, z_{j}, p_{i j}\right)$. Let $g_{1} \ldots, g_{m}$ be $m$ functions in $M_{U}$ such that

at every point $\bar{P}$ in $U$.

$$
g_{j}(\bar{P})=\left(\frac{\partial}{\partial z_{j}}\right)_{P}
$$

It is easy to check that the $g_{j}^{\prime} s$ are a basis of $M_{U}$ and, thus, every element $f$ in $M_{U}$ can be written as:

where the $f_{j}^{\prime} s$ belong to $A_{\vec{B}}$.

$$
f=\sum_{j} f_{j} g_{j}
$$

We shall write from now on:

instead of $f=\sum_{j} f_{j} g_{j}$.

$$
f=\sum_{j} f_{j} \frac{\partial}{\partial z_{j}}
$$

If $h$ is a function in $M^{*}$, we shall write

$$
h=\sum_{j} h_{j} d z_{j} .
$$

From the former Lemma it follows immediately that every $M$-valued $p$-covariant, $q$-contravariant tensor can be written as

$$
T_{p}^{q}=\sum_{j}\left(T_{p}^{q}\right)_{j} \frac{\partial}{\partial z_{j}}
$$

where the $\left(T_{p}^{q}\right)_{j}^{\prime} s$ are ordinary tensors on $\bar{B}$ such that

$$
T_{p}^{q}\left(\bar{D}_{1} \ldots, \bar{D}_{p}, \bar{\omega}_{1} \ldots, \bar{\omega}_{q}\right)=\sum_{j}\left[\left(T_{p}^{q}\right)_{j}\left(\bar{D}_{1} \ldots, \bar{D}_{p}, \bar{\omega}_{1} \ldots, \bar{\omega}_{q}\right)\right] \frac{\partial}{\partial z_{j}}
$$

for any $\left(\bar{D}_{1} \ldots, \bar{D}_{p}, \bar{\omega}_{1} \ldots, \bar{\omega}_{q}\right)$.

For instante, the structure from $\theta$ can be written as

$$
\theta=\sum_{j}\left(d z_{j}-\sum_{i} p_{i j} d x_{i}\right) \frac{\partial}{\partial z_{j}}
$$

as we proved in theorem 1.1.

Finally, we will calculate the derivative of an arbitrary function $f$ in $M$ with respect to a derivation $\bar{D}$ on $\bar{B}$ with the derivation law given by Definition 2.8:

$$
\bar{D} f=\bar{D}\left(\sum_{j} f_{j} \frac{\partial}{\partial z_{j}}\right)=\sum_{j}\left(\bar{D} f_{j}\right) \frac{\partial}{\partial z_{j}}+\sum_{j} f_{j}\left(\bar{D} \frac{\partial}{\partial z_{j}}\right) .
$$

By definition 2.8. we have:

if

$$
d z_{k}\left(\bar{D} \frac{\partial}{\partial z_{j}}\right)=-\left(D^{\prime \prime \nabla} d z_{k}\right) \frac{\partial}{\partial z_{j}}=\sum_{i} \mu_{i} \Gamma_{i j}^{k}
$$

$3 *$

$$
D^{\prime \prime}=\sum_{i} \mu_{i} \frac{\partial}{\partial z_{i}} \quad \text { and } \quad \frac{\partial \nabla}{\partial z_{i}} d z_{k}=-\sum_{j} \Gamma_{i j}^{k} d z_{j}
$$


where $\Gamma_{i j}^{k}$ are the components of the connection in the open set induced by $U$ in $F$.

So,

$$
\bar{D} f=\sum_{j}\left(\bar{D} f_{j}\right) \frac{\partial}{\partial z_{j}}+\sum_{i, j, k} f_{j} \mu_{i} \Gamma_{i j}^{k} \frac{\partial}{\partial z_{k}} .
$$

Notation. In order to avoid any confusion between the ordinary tensor and contracted products and the corresponding generalized products we have introduced in this paper, we shall denote the latter ones by the notation (o).

\section{Generalized Infinitesimal Contact Transformations}

We shall say that a vector field $\bar{D}$ on $\bar{B}$ is a generalized infinitesimal contact transformation (g.i.c.t.) when for every derivation law $V$ on $M$ we have

$$
L_{\bar{D}} \theta=\Phi \circ \theta
$$

where $\Phi \in \underset{A_{\bar{B}}}{\operatorname{Hom}}(M, M)$, the dot product $\circ$ is the one induced by the usual bilinear product $\operatorname{Hom}_{A_{\bar{B}}}(M, M) \times M \rightarrow M$ and $L_{D}$ is taken with respect to $\nabla$.

It is easy to prove that a vector field $\bar{D}$ on $\bar{B}$ is a g.i.c.t. if the former condition is verified for a derivation law $\nabla$; i.e. if one law verifies the condition, all of them do.

Theorem 3.1. Given a vector field $D$ on the manifold $B$, there is one and only one g.i.c.t. $\bar{D}$ such that $d \bar{\pi}(\bar{D})=D$.

Proof. It suffices to prove that the system of equations $L_{\bar{D}} \theta=\Phi \circ \theta$ and $d \bar{\pi}(\bar{D})=D$, with unknowns $\bar{D}, \Phi$ has a unique solution in $\bar{\pi}^{-1}(U)$, where $U$ is the open set in $B$ where the vector field $D$ is defined.

Let $U^{\prime}$ be an open set in $\bar{\pi}^{-1}(U)$ with local canonical coordinates $\left(x_{i}, z_{j}, p_{i j}\right)$; we have then:

$$
\begin{aligned}
L_{\bar{D}} \theta & =L_{\bar{D}}\left(\sum_{j} \theta_{j} \frac{\partial}{\partial z_{j}}\right)=\sum_{j}\left[\left(L_{\bar{D}} \theta_{j}\right) \frac{\partial}{\partial z_{j}}+\theta_{j}\left(L_{\bar{D}} \frac{\partial}{\partial z_{j}}\right)\right] \\
& =\sum_{j}\left(L_{\bar{D}} \theta_{j}+\sum_{h} a_{j h} \theta_{h}\right) \frac{\partial}{\partial z_{j}} .
\end{aligned}
$$

Hence, the condition $L_{\bar{D}} \theta_{j}=\sum_{h} b_{j h} \theta_{h}$, where the $b_{j h}$ 's belong to the ring of $C^{\infty}$ class function defined on $U^{\prime}$. On the other hand, if $d \bar{\pi}(\bar{D})=D$, we have

whenever

$$
\bar{D}=\lambda_{i j} \frac{\partial}{\partial p_{i j}}+\mu_{i} \frac{\partial}{\partial z_{i}}+v_{j} \frac{\partial}{\partial x_{j}}
$$

$$
D=\mu_{i} \frac{\partial}{\partial z_{i}}+v_{j} \frac{\partial}{\partial x_{j}},
$$


so :

$$
\begin{aligned}
& b_{j h}=\frac{\partial \mu_{j}}{\partial z_{h}}-\sum_{i} p_{i j} \frac{\partial v_{i}}{\partial z_{h}} \\
& \lambda_{i j}=\frac{\partial \mu_{j}}{\partial x_{i}}-\sum_{k} p_{k j} \frac{\partial v_{k}}{\partial x_{i}}+\sum_{h} b_{j h} p_{i h} .
\end{aligned}
$$

This proves that there exists on $U^{\prime}$ a unique solution $\left(\bar{D}_{U^{\prime}}, \Phi_{U^{\prime}}\right)$ of the system of equations

$$
L_{\bar{D}} \theta=\Phi \circ \theta ; \quad d \bar{\pi}(\bar{D})=D
$$

Let now $\bar{D}$ and $\Phi$ be the unique vector field and homomorphism that induce $\bar{D}_{U^{\prime}}$ and $\Phi_{U^{\prime}}$ on $U^{\prime}$, for every $U^{\prime} \subset \bar{\pi}^{-1}(U)$ of the former type. Then, $(\bar{D}, \Phi)$ is the unique solution on $\bar{\pi}^{-1}(U)$ of our system of equations.

Definition 3.2. $\bar{D}$ is called canonical lift of $D$ to $\bar{B}$.

The canonical lifts of the vector fields on $B$ that $d \pi$ projects on the zero derivation of $V$ are of main importance; these g.i.c.t. are called vertical.

\section{Variational Theory on the Vertical Bundle $\bar{B}$}

The notion of a field in the sense of a physical system defined by a given lagrangian can be formalized in the following way:

Let $V$ be a differentiable $n$-dimensional manifold such as those we have dealt with in the former sections, and assume, furthermore, that a non vanishing $n$-form $\omega$, called volume element, is defined on every point of $V$ and let $F$ be a $m$-dimensional differentiable manifold.

Definition 4.1. A real-valued differentiable function $\mathscr{L}$ on the vertical bundle $\bar{B}$ is called a $F$-type field on $V . V, B, \bar{B}, \mathscr{L}$ are respectively called base space, configuration space, state space and lagrangian of the field.

Starting from this definition we can state a variational problem on $\bar{B}$ in the following way:

The volume element $\omega$ of $V$ can be mapped into the algebra of forms on $\bar{B}$ by means of the injection $(\pi \cdot \bar{\pi})^{*}$ (transposed of the projection $\left.\pi \cdot \bar{\pi}\right)$. Then, it makes sense to consider the $n$-form $\mathscr{L} \omega$ on $\bar{B}$ and define a real-valued function $\mathcal{L}$ on the set $S$ of regular cross sections $s$ of $B$ as follows :

$$
\mathfrak{L}(s)=\int_{\bar{s}(V)} \mathscr{L} \omega
$$

where $\bar{s}$ is the canonical lift of $s$ to $\bar{B}$.

If $D$ is a differentiable vector field on $B$, we can introduce the notion of derivative $D \mathfrak{Q}$ of the function $\mathfrak{Q}$ with respect to the field $D$ as the function on $S$ defined by:

$$
D_{s} \mathfrak{I}=\int_{\bar{s}(V)} L_{\bar{D}}(\mathscr{L} \omega)
$$

where $\bar{D}$ is the canonical lift of $D$ to $\bar{B}$. 
Finally, we give a notion of stationariedness as follows: For every section $s$ in $S$, let $(\delta \mathscr{S})_{s}$ be the linear function on the vector space $\{D\}$ of the vector fields with compact support in $B$ defined by:

$$
(\delta \mathfrak{L})_{s} D=D_{s} \mathfrak{I} \text {. }
$$

Definition 4.2. $A$ regular cross section $s$ of $B$ is stationary when $(\delta \Omega)_{s}=0$.

The basic problem is now to characterize the stationary transversal sections as solutions of some type of differential equations. We shall see later (and this is our main result) that to each linear connection $\nabla$ on the differentiable $F$ manifold (with the symmetry condition $D_{1}^{\nabla} D_{2}=D_{2}^{\nabla} D_{1}$ ) can be canonically associated a system of modulevalued differential forms of different degree on the vertical bundle $\bar{B}$, whose solutions are the stationary transversal sections of our variational problems. This system of differential forms will be called field equations, in a slightly abusive language, since these equations do not only depend on the considered field but also on the initial connection.

With this consideration in mind let us now fix once and for all the connection $\nabla$ and assume that all differentiation operations will be done with respect to the derivation law that $\nabla$ induces on the $A_{\bar{B}}$-module $M$ in the sense of the Definition 2.8.

The basic tools used to obtain these equations are a function $f$ and an $(n-1)$-form $\Omega$, both defined on $\bar{B}$ and $M^{*}$-valued. On the analogy of analytic dynamics - which is the one-dimensional manifold case (timeline) in our scheme - they will be called virtual work function ${ }^{1}$ and momentum $(n-1)$-form on the field we are dealing with.

Theorem 4.1. One and only one function $f$ and one and only one $(n-1)$ form $\Omega$ on $\bar{B}$, can be found such that:

a) $i X \circ \omega=\Omega$

b) $i X \circ d \theta+d \mathscr{L}=f \circ \theta$

where $X$ is a 1-contravariant $M^{*}$-valued tensor on $\bar{B}$ and products in a) and $b)$ are taken with respect to bilinear products $M^{*} \times A_{\vec{B}} \rightarrow M^{*}$ and $M^{*} \times M \rightarrow A_{\bar{B}}$ defined by the module structure and by duality notion, respectively.

Proof. Let $U$ be any open set in $\bar{B}$ with local canonical coordinates, $\left(x_{i}, z_{j}, p_{i j}\right)$. The map induced by $d \theta$ on $U$ is :

$$
\begin{aligned}
d \theta & =d\left(\sum_{j} \theta_{j} \frac{\partial}{\partial z_{j}}\right)=\sum_{j}\left(d \theta_{j} \frac{\partial}{\partial z_{j}}-\theta_{j} \wedge d \frac{\partial}{\partial z_{j}}\right) \\
& =\sum_{j} d \theta_{j} \frac{\partial}{\partial z_{j}}-\sum_{j} \theta_{j} \wedge \sum_{h k}\left(\Gamma_{h j}^{k} d z_{h}\right) \frac{\partial}{\partial z_{k}} \\
& =\sum_{k}\left(d \theta_{k}-\sum_{h j} \Gamma_{h j}^{k_{j}} \theta_{j} \wedge d z_{h}\right) \frac{\partial}{\partial z_{k}}
\end{aligned}
$$

${ }_{1}$ Since in analytic dynamics $f$ is the element in $M^{*}$ that assigns the virtual work form, $\left(\omega^{T}\right)_{P}$ to every point $\bar{P}$ in $\bar{B}$. 
where the functions $\Gamma_{h j}^{k}$ are the components of the connection in the open set induced by $U$ in $F$. have:

If $X_{U}=\sum_{j} X_{j} d z_{j}$ is a 1-contravariant $M_{U}^{*}$-valued tensor on $U$, we

$$
i X_{U} \circ d \theta=\sum_{k}\left(i X_{k} \cdot d \theta_{k}-\sum_{h j} \Gamma_{h j}^{k}\left[\theta_{j}\left(X_{k}\right) d z_{h}-\left(X_{k} z_{h}\right) \theta_{j}\right]\right) .
$$

On the other hand, if $f_{U}=\sum_{j} f_{j} d z_{j}$ is an element in $M_{U}^{*}$, we have:

$$
f_{U} \circ \theta=\sum_{j} f_{j} \theta_{j}
$$

Raplacing now $i X_{U} \circ d \theta$ and $f_{U} \circ \theta$ in b) and putting $\Omega_{U}=i X_{U} \circ \omega$, we obtain a local expression in $U$ of the system of tensor equations a) and b) with unknowns $X_{U}, f_{U}$ and $\Omega_{U}$.

Applying b) to the vector fields $\frac{\partial}{\partial p_{i j}}$ and $\frac{\partial}{\partial z_{j}}$ succesively, we have:

$$
X_{j} x_{i}=-\frac{\partial \mathscr{L}}{\partial p_{i j}} ; \quad f_{j}=\frac{\partial \mathscr{L}}{\partial z_{j}}-\sum_{i h k} p_{i h} \Gamma_{j h}^{k} \frac{\partial \mathscr{L}}{\partial p_{i k}} .
$$

Furthermore, applying b) once more to the vector fields $\frac{\partial}{\partial x_{i}}$, we obtain a solvable system of equations with the rest of the unknowns. It follows then that the system of equations a) and b) determines $f_{U}$ and $\Omega_{U}$, since $X_{j} x_{i}$ determine $\Omega_{U}$.

If we call $f$ and $\Omega$ the function and the $(n-1)$-form on $\bar{B}$ such that the maps induced by them on every open set $U$ of the former type are $f_{U}$ and $\Omega_{U}$, respectively, then $f$ is the only function and $\Omega$ the only form verifying the conditions of the theorem.

Remark. On account of the construction we have followed, the $(n-1)$ momentum form $\Omega$ of the field does not depend on the connection $\nabla$.

\section{Fundamental Formula of Variation}

The formula we state next is the basic tool we use in proving the main theorems in this paper.

Theorem 5.1. Let $s$ be a regular cross section and $D$ a vector field on the bundle $B, \bar{s}$ and $\bar{D}$ the canonical lifts of $s$ and $D$ to $\bar{B}$, and let $f$ and $\Omega$ be the virtual work function and the momentum form of the field. Then, on $\bar{s}(V)$ we have:

$$
L_{\bar{D}}(\mathscr{L} \omega)=\theta(\bar{D}) \circ(d \Omega+f \circ \omega)-d(\theta(\bar{D}) \circ \Omega-i \bar{D} . \mathscr{L} \omega)
$$

where the tensor products are taken with respect to the bilinear products $M \times M^{*} \rightarrow A_{\bar{B}}$ and $A_{\bar{B}} \times M^{*} \rightarrow M^{*}$.

Proof. First we give the proof for the case $d \pi(D)=0$.

Then $\bar{D}$ is a vertical g.i.c.t. and we have

$$
L_{\bar{D}}(\mathscr{L} \omega)=(\bar{D} \mathscr{L}) \omega=(d \mathscr{L}) \bar{D} \omega
$$


Applying b) of Theorem 4.1., we have:

$$
(i X \circ d \theta) \bar{D}+d \mathscr{L}(\bar{D})=(f \circ \theta) \bar{D}
$$

Moreover, since $\bar{D}$ is a g.i.c.t.

$$
L_{\bar{D}} \theta=i \bar{D} \circ d \theta+d(\theta(\bar{D}))=\Phi \circ \theta
$$

and taking the inner product of $\left(1^{\prime}\right)$ and $X$, we have:

$$
i X \circ(i \bar{D} \circ d \theta)+i X \circ d(\theta(\bar{D}))=i X \circ(\Phi \circ \theta)
$$

Adding up (1) and (2), it follows from

$$
i X \circ(i \bar{D} \circ d \theta)=-i \bar{D} \cdot(i X \circ d \theta) \text {, }
$$

that

$$
(d \mathscr{L}) \bar{D}=-i X \circ d(\theta(\bar{D}))+(f \circ \theta) \bar{D}+i X \circ(\Phi \circ \theta)
$$

and multiplying by the volume element, we have

$$
L_{\bar{D}}(\mathscr{L} \omega)=-[i X \circ d(\theta(\bar{D}))] \omega+(f \circ \theta) \bar{D} \omega+(i X \circ(\Phi \circ \theta)) \omega .
$$

We will now obtain another expression for the term $[i X \circ d(\theta(\bar{D}))] \omega$ proceeding as follows:

First take the exterior product by $\omega$ on both sides of $i \bar{D} \circ d \theta$ $+d(\theta(\bar{D}))=\Phi \circ \theta$; we have that $d(\theta(\bar{D})) \wedge \omega=\Phi \circ \theta \wedge \omega$, since $i \bar{D} \circ d \theta \wedge \omega=0$ as we shall see later. Taking $i X \circ$ on both sides again we have, by a) in Theorem 4.1., that

$$
[i X \circ d(\theta(\bar{D}))] \omega-d(\theta(\bar{D})) \wedge \Omega=i X \circ(\Phi \circ \theta \wedge \omega) .
$$

Applying now the exterior differential and inner product formulae for a product, and substituting in (3) we have:

$$
L_{\bar{D}}(\mathscr{L} \omega)=\theta(\bar{D}) \circ(d \Omega+f \circ \omega)-d(\theta(\bar{D}) \circ \Omega)+\Phi \circ \theta \wedge \omega
$$

If we specialize (4) to $\bar{s}(V)$ the last term vanishes by the definition of the canonical lift $\bar{s}$ of $s$ to $\bar{B}$. This proves the case $d \pi(D)=0$.

Before proving the general case, let us check the identity $i \bar{D} \circ d \theta \wedge \omega=0$. It suffices to prove it for an arbitrary neighbourhood $U$ with canonical local coordinates $\left(x_{i}, z_{j}, p_{i j}\right)$. From the symmetry $\Gamma_{h j}^{k}=\Gamma_{j h}^{k}$ we have:

$$
\begin{aligned}
i \bar{D} \circ d \theta \wedge \omega & =\sum_{k}\left\{\left[i \bar{D} \cdot d \theta_{k}-\sum_{h j} \Gamma_{h j}^{k}\left(i \bar{D} \cdot \theta_{j} d z_{h}-\bar{D} z_{h} \theta_{j}\right)\right] \wedge \omega\right\} \frac{\partial}{\partial z_{k}} \\
& =\sum_{k}\left[\sum_{h j} \Gamma_{h j}^{k}\left(\bar{D} z_{j} d z_{h}-\bar{D} z_{h} d z_{j}\right) \wedge \omega\right] \frac{\partial}{\partial z_{k}}=0 .
\end{aligned}
$$

Let us turn now to the general case. We have:

$$
L_{\bar{D}}(\mathscr{L} \omega)=(\bar{D} \mathscr{L}) \omega+\mathscr{L} L_{\bar{D}} \omega .
$$

The vector field $D$ can be written as a sum $D=D_{1}+D_{2}$ of two vector fields $D_{1}, D_{2}$ such that $d \pi\left(D_{1}\right)=0$ and such that $D_{2}$ induces a vector 
field on $s(V)$. Then, if $\bar{D}_{1}, \bar{D}_{2}$ are the canonical lifts of $D_{1}, D_{2}$ to $\bar{B}$, we have $\bar{D}=\bar{D}_{1}+\bar{D}_{2}$, where $\bar{D}_{1}$ is a vertical g.i.c.t. and $\bar{D}_{2}$ induces a vector field on $\bar{s}(V)$. In these conditions we have

$$
L_{\bar{D}}(\mathscr{L} \omega)=\left(\bar{D}_{1} \mathscr{L}\right) \omega+\left(\bar{D}_{2} \mathscr{L}\right) \omega+\mathscr{L} L_{\bar{D}_{2}} \omega
$$

Applying the result (4) to $\bar{D}_{1}$, we have:

$$
\left(\bar{D}_{1} \mathscr{L}\right) \omega=\theta\left(\bar{D}_{1}\right) \circ(d \Omega+f \circ \omega)-d\left(\theta\left(\bar{D}_{1}\right) \circ \Omega\right)+\Phi_{1} \circ \theta \wedge \Omega
$$

Furthermore,

$$
\left(\bar{D}_{2} \mathscr{L}\right) \omega+\mathscr{L} L_{\bar{D}_{2}} \omega=L_{\bar{D}_{2}}(\mathscr{L} \omega)=i \bar{D}_{2} \cdot d(\mathscr{L} \omega)+d\left(i \bar{D}_{2} \cdot \mathscr{L} \omega\right)
$$

So, finally, we have:

$$
\begin{gathered}
L_{\bar{D}}(\mathscr{L} \omega)=\theta\left(\bar{D}_{1}\right) \circ(d \Omega+f \circ \omega) \\
-d\left(\theta\left(\bar{D}_{1}\right)-i \bar{D}_{2} \cdot \mathscr{L} \omega\right)+\Phi_{1} \circ \theta \wedge \Omega \\
+i \bar{D}_{2} \cdot d(\mathscr{L} \omega)
\end{gathered}
$$

$\bar{D}_{1}$ is vertical, so $i \bar{D}_{2} . \mathscr{L} \omega=i \bar{D}$. $\mathscr{L} \omega$. Moreover, formula (5) induces on $\bar{s}(V)$ :

$$
L_{\bar{D}}(\mathscr{L} \omega)=\theta\left(\bar{D}_{1}\right) \circ(d \Omega+f \circ \omega)-d\left(\theta\left(\bar{D}_{1}\right)-i \bar{D} \cdot \mathscr{L} \omega\right),
$$

since $\Phi_{1} \circ \theta \wedge \Omega$ is zero by the definition of $\bar{s}$, and $i \bar{D}_{2} \cdot d(\mathscr{L} \omega)$ vanishes too, since $D_{2}$ is tangent to $s(V)$. This completes the proof.

\section{Field Equations and Noether Invariants on the Vertical Bundle $\bar{B}$}

Let a field of the type defined by a differentiable manifold $F$ with a Lagrangian $\mathscr{L}$ be given on a differentiable manifold $V$ with volume element $\omega$. The system of differential froms $\{\theta, d \Omega+f \circ \omega\}$ on the vertical bundle $\bar{B}$, where $\theta$ is the structure 1 -form on $\bar{B}$ and $f, \Omega$ are the virtual work function and the momentum $(n-1)$-form of the field, are called field equations.

The stationary sections in the sense of Definition 4.2. can now be characterized as follows:

Theorem 6.1. The stationary cross sections $s$ on the bundle $B$ are the projections by $\bar{\pi}$ of the cross sections $\bar{s}$ on $\bar{B}$ - regarded as a bundle on $V-$ which are solutions of the field equations $\{\theta, d \Omega+f \circ \omega\}$.

Proof. Let $s$ be a stationary section and $\bar{s}$ its canonical lift to $\bar{B}$. We prove that $\bar{s}(V)$ - which is a solution of $\theta$ by definition - is a solution of $d \Omega+f \circ \omega$ too.

Indeed, let $U$ be an open set in $\bar{B}$ with local canonical coordinates $\left(x_{i}, z_{j}, p_{i j}\right)$ such that $U \cap \bar{s}(V) \neq \emptyset$. If $D_{i}=d \bar{s}\left(\frac{\partial}{\partial x_{i}}\right)$, then $(d \Omega+f \circ \omega)$ $\left(D_{1}, \ldots, D_{n}\right)=\sum_{j} \varphi_{j} d z_{j}$, where the $\varphi_{j}^{\prime} s$ are differentiable functions on the submanifold $U \cap \bar{s}(V)$. Let now $C$ be a differentiable $n$-dimensional chain in $\pi \cdot \bar{\pi}(U)$, and $D$ any vector field on $B$ with compact support $K$, 
such that $\bar{\pi}^{-1}(K) \cap \bar{s}(V) \subset \bar{s}(C)$ and $\bar{\pi}^{-1}(K) \cap \bar{s}(\partial C)=\emptyset$, we denote $\partial C$ the boundary of $C$. Applying the fundamental variation formula, we have:

$$
\begin{aligned}
0 & =(\delta \mathscr{L})_{s} D=\int_{\bar{s}(V)} L_{\bar{D}}(\mathscr{L} \omega)=\int_{\bar{s}(C)} L_{\bar{D})}(\mathscr{L} \omega) \\
& =\int_{\bar{s}(C)} \theta(\bar{I}) \circ(d \Omega+f \circ \omega)=\int_{\bar{s}(C)} \sum_{j} \theta_{j}(\bar{D}) \varphi_{j} \omega
\end{aligned}
$$

Using the fact that $D$ is arbitrary, the $\varphi_{j}^{\prime} s$ vanish at the inner points of $\bar{s}(C)$, hence $d \Omega+f \circ \omega$ vanishes at the inner points of $\bar{s}(C), U$ being arbitrary, we get the intended result $(d \Omega+f \circ \omega)_{\bar{s}(V)}=0$.

Conversely, let $\bar{s}$ be a cross section on $\bar{B}$ - regarded as a bundle on $V$ and assume that $\bar{s}$ is a solution of the field equations, that is the canonical lift of $s$ to $\bar{B}$ is a solution of the $n$-form $d \Omega+f \circ \omega$; let $D$ be any vector field on $B$ with compact support $K$, and $C$ a differentiable $n$-dimensional chain in $V$ with the same conditions we stated before. Applying the fundamental variation formula once more, we have:

$(\delta \mathscr{L})_{s} D=\int_{\bar{s}(V)} L_{\bar{D}}(\mathscr{L} \omega)=\int_{\bar{s}(C)} L_{\bar{D}}(\mathscr{L} \omega)=\int_{\bar{s}(C)} \theta(\bar{D}) \circ(d \Omega+f \circ \omega)=0$

and using now the fact that $D$ is arbitrary, we obtain $(\delta \mathscr{I})_{s}=0$, so $s$ is stationary.

If $U$ is an open set in $\bar{B}$ with local canonical coordinates $\left(x_{i}, z_{j}, p_{i j}\right)$, a simple local calculus shows that the field equations turn into

$\left\{\sum_{j} \theta_{j} \frac{\partial}{\partial z_{j}}, \sum_{j}\left(d \Omega_{j}+(-1)^{n} \sum_{h k} \Gamma_{h j}^{k} \Omega_{k} \wedge d z_{h}+f_{j} \mathscr{J} d x_{1} \wedge \ldots \wedge d x_{n}\right) d z_{j}\right\}$ where

$$
\begin{aligned}
\theta_{j} & =d z_{j}-\sum_{i} p_{i j} d x_{i} \\
f_{j} & =\frac{\partial \mathscr{L}}{\partial z_{j}}-\sum_{l i m} \Gamma_{j m}^{k} p_{l m} \frac{\partial \mathscr{L}}{\partial p_{l k}} \\
\Omega_{j} & =\sum_{i}(-1)^{i} \frac{\partial \mathscr{L}}{\partial p_{i j}} d x_{1} \wedge \ldots \wedge \widehat{d x_{i}} \wedge \ldots \wedge d x_{n} \\
\mathscr{I} & =\omega\left(\frac{\partial}{\partial x_{1}}, \ldots, \frac{\partial}{\partial x_{n}}\right) .
\end{aligned}
$$

This leads immediately to the following.

Corollary (Euler-Lagrange Equations). A necessary and sufficient condition for a cross section $z_{j}=z_{j}\left(x_{i}\right)$ to be stationary is:

$$
p_{i j}=\frac{\partial z_{j}}{\partial x_{i}} ; \quad \frac{\partial \mathscr{L}}{\partial z_{j}}-\sum_{j} \frac{\partial}{\partial x_{i}}\left(\frac{\partial \mathscr{L}}{\partial p_{i j}}\right)-\frac{1}{\mathscr{J}} \sum_{i} \frac{\partial \mathscr{J}}{\partial x_{i}} \frac{\partial \mathscr{L}}{\partial p_{i j}}=0 .
$$

Proof. It suffices to write that the restriction to the canonical lift of $z_{j}=z_{j}\left(x_{i}\right)$ of the second differential form of the field equations vanishes, and to take into account the symmetry property $\Gamma_{i j}^{h}=\Gamma_{j i}^{h}$ of the coefficients of the connection. 
We finish this section formulating in this new scheme the notion of an invariant variational problem by a Lie group, and stating E. Noether's theorem for thes invariant problems.

Let $G$ be a Lie group acting on $B$ by means of fibre preserving automorphisms of $B$, i.e. fibres are turned into fibres. Every element $a$ of the Lie algebra $A_{G}$ of $G$ defines a vector field $D_{a}$ on $B$ in a well-known way. If $\bar{D}_{a}$ is the canonical lift of $D_{a}$ to $\bar{B}$, we have a linear mapping of $A_{G_{*}}$ into the space of the vector fields on $\bar{B}$.

$$
a \in A_{G} \rightarrow \bar{D}_{a} .
$$

Definition 6.1. A field with lagrangian $\mathscr{L}$ is said to be invariant by a Lie group $G$ acting on the bundle $B$ in the former way when, for every element $a$ in its Lie algebra $A_{G}$, an $(n-1)$-form $\omega_{a}$ exists such that $L_{\bar{D}_{a}}(\mathscr{L} \omega)$ $=d \omega_{a}$.

Given an invariant (in our sense) field, let $\tau_{\xi}$ be the mapping of the Lie algebra $A_{G}$ to the algebra of differential forms on $\bar{B}$ defined as follows:

$$
\tau_{G}: a \in A_{G} \rightarrow \theta\left(\bar{D}_{a}\right) \circ \Omega-i \bar{D}_{a} \cdot \mathscr{L} \omega-\omega_{a}
$$

Applying the fundamental formula of variation, it follows immediately that:

Theorem 6.2. (E. Noether). For every stationary cross section $s$ on the bundle $B$ and every element $a$ in the Lie algebra $A_{G}$ we have:

$$
\left(d \tau_{G}(a)\right)_{\bar{s}(V)}=0 \text {. }
$$

Or, for every $n$-dimensional differentiable chain $C$ in $V$, we have:

$$
\int_{\bar{s}(\partial C)} \tau_{G(r}(a)=0 .
$$

The values taken by the mapping $\tau_{G}$ are (in our terminology) the so-called Noether invariants in classical variation calculus.

Remark. Since the $(n-1)$-form $\Omega$ does not depend on the connection $\nabla$ the Noether invariants do not depend on it either.

\section{References}

1. Jost, R.: Poisson brackets (An unpedagogical lecture). Rev. Mod. Phys. 36, 572 (1964).

2. Koszul, J. L.: Lectures on fibre bundles and differential geometry. Bombay: Institute of Fundamental Research 1960.

3. LANG, S.: Introduction to differentiable manifolds. New York: Wiley 1962.

4. Lichnerowicz, A.: Propagateurs et Commutateurs en Relativité Général. Paris, Publications mathématiques de l'Institut des Hautes Etudes Scientifiques. No. 10, 1961.

5. Mackey, G. W.: Mathematical foundations of quantum mechanics. New York: Benjamin 1963. 
44 P. L. GARCíA et al.: Symplectic Approach to the Theory of Quantized Fields

6. Segal, I. E.: Differential operators in the manifold of solutions of a non-linear differential equation. J. Math. 54, 71 (1965).

7. - Quantization of non-linear systems. J. Math. Phys. 1, 6, 468 (1960).

8. - La Variété des Solutions d'une Equation Hyperbolique, Nonlinear d'Ordre 2. Séminaire sur les equations aux derivées partielles III. Collège de France $1964 / 65$.

9. Trautman, A.: Noether equations and conservation laws. - Commun. Math. Phys. 6, 248 (1967).

P. L. García, A. Pérez-Rendón

Departamento de Algebra y Fundamento Facultad de Ciencias

Universidad de Barcelona

Barcelona, España 